\title{
Vitiligo - Part 2 - classification, histopathology and treatment ${ }^{*}$
}

\author{
Adriane Reichert Faria ${ }^{1,2}$ \\ Marcelo Távora Mira²
}

\author{
Roberto Gomes Tarlé ${ }^{1}$
}

Gerson Dellatorre ${ }^{1}$

DOI: http://dx.doi.org/10.1590/abd1806-4841.20142717

\begin{abstract}
In an unprecedented effort in the field of vitiligo, a global consensus resulted on a suggested new classification protocol for the disease. The main histopathological finding in vitiligo is the total absence of functioning melanocytes in the lesions, while the inflammatory cells most commonly found on the edges of the lesions are CD4+ and CD8+ T lymphocytes. Physical and pharmacological treatment strategies aim to control the autoimmune damage and stimulate melanocyte migration from the unaffected edges of lesions and the outer hair follicle root sheath to the affected skin; moreover, surgical treatments can be combined with topical and physical treatments.
\end{abstract}

Keywords: Histology; Therapeutics; Treatment outcome; Vitiligo

\section{VITILIGO CLASSIFICATION}

According to the review conducted by the Vitiligo Global Issues Consensus Conference (1) between 2011-2012, vitiligo can be classified in the following clinical forms (Table 1):

- Non-segmental vitiligo (NSV): a group that comprises acrofacial, mucosal, generalized or common, universal, and mixed forms besides rare forms.

a. Acrofacial: it can affect, face, head, hands and feet, and preferably involve the perioral region and the extremities of digits;

b. Mucosal: affects the oral and genital mucosae. Furthermore, areas of mucosa may also be affected in patients with acrofacial, common, or universal forms; when it involves only one mucosal site it is classified as indeterminate; ${ }^{1}$

c. Generalized or common (Figure 1): Macules / patches are often symmetrical; it can affect any part of the tegument, mainly hands, fingers, face and trauma-exposed areas.

d. Universal: is the form that affects the largest extent of tegument (80-90\% of body surface), and it is the most common form in adulthood. The generalized or common form usually precedes it. e. Mixed: it is the concomitant involvement of segmental and non-segmental vitiligo. Most often, the segmental form precedes NSV.

f. Rare forms: vitiligo punctata, minor and follicular. These types were also considered unclassifiable.
- Segmental Vitiligo: it can affect one, two or multiple segments (Figure 2). The unisegmental form is the most common one and consists of one or more white macules on one side of the body, usually respecting the body midline, and there is also involvement of body hair (leukotrichia) besides rapid onset of the condition. Less commonly, it can affect two or more segments and even have bilateral segmental distribution, starting simultaneously or not.

- Unclassifiable forms or undetermined vitiligo a) Focal: Isolated white macule without segmental distribution. This form can evolve to segmental or NSV forms.

b) Mucosal: when only one mucosa is affected.

This consensus was a major advance towards the standardized classification of vitiligo, but there are still some aspects that need to be better appreciated in a forthcoming review, such as: 1) the text about the type formerly known as vulgaris was ambiguous since the table mentioned it as a generalized form and in the appendix, it appears as common; 2) acrofacial vitiligo can also have genital lesions, which was not described in the classification section. ${ }^{2}$ Furthermore, one can not affirm that acrofacial vitiligo will necessarily evolve to more severe forms, so prospective studies and cohort studies are needed to investigate this hypothesis; 3 ) rare forms, which were designated as unclassifiable, 
TABLE 1: Vitiligo Classification

\begin{tabular}{ll}
\hline Types & Subtypes \\
\hline Non-segmental vitiligo & Acrofacial \\
& Mucosal (more than one site affected) \\
& Generalized or Common \\
& Universal \\
& Mixed (associated with segmental vitiligo) \\
& Rare forms \\
Segmental & Unisegmental, bisegmental or multisegmental \\
Unclassified or indeterminate & Focal \\
& Mucosal (only one site affected) \\
\hline
\end{tabular}

Adapted from: Revised classification/nomenclature of vitiligo and related issues: the Vitiligo Global Issues Consensus Conference - 2012.

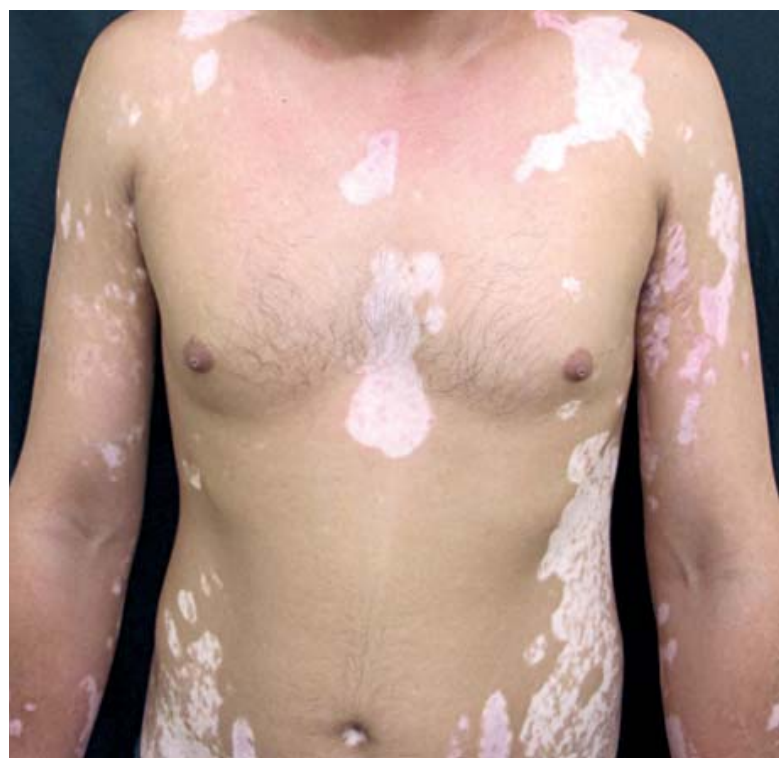

Figure 1: Common Vitiligo. Bilateral and often symmetrical lesions characterize common vitiligo

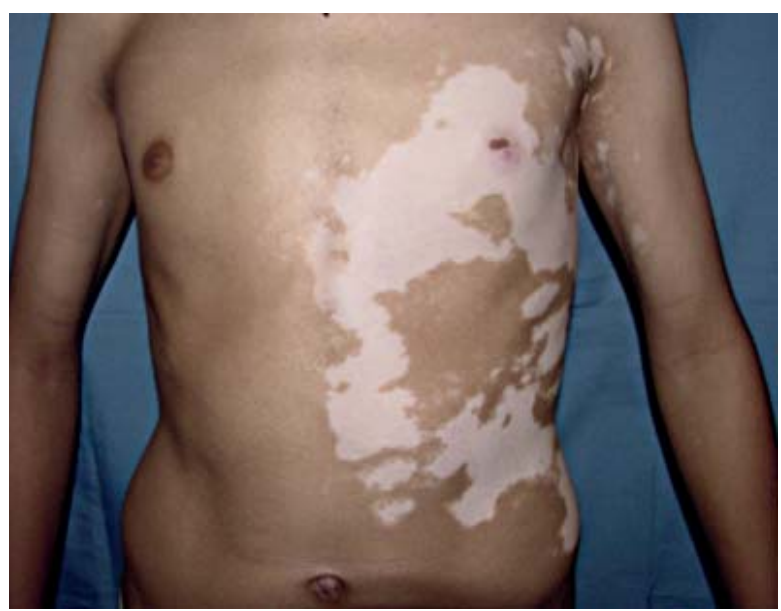

FigurE 2: Segmental Vitiligo. Unilateral lesions that respect, almost completely, the body midline, characterize segmental vitiligo. There are rare cases of bilateral segmental vitiligo should not be under the NSV group in the table but in the unclassified group.

\section{HISTOPATHOLOGY}

Melanocytes are derived from neural crest cells. Neurons, glial cells, cardiac cells, craniofacial tissue and adrenal medulla are also originated from such pluripotent cells. Melanocyte precursors, known as melanoblasts, migrate, proliferate and differentiate en route to their destination in the basal epidermis and hair follicles. ${ }^{3}$

Epidermal melanocytes and keratinocytes form structural and functional units, known as epidermal melanin units, in which every melanocyte carries its melanosomes through dendrites to approximately 36 associated keratinocytes. Skin pigmentation results of this close interaction between melanocytes that produce melanosomes and keratinocytes that receive them. ${ }^{4}$ Melanocytes are located in the basal layer of the epidermis at a ratio of one to every 5 basal keratinocytes. ${ }^{5,6}$

The maintenance of this balance occurs through controlled induction of melanocyte division. ${ }^{7}$ To proliferate, the melanocyte will detach from the basement membrane and from keratinocytes, then retract its dendrites, divide, migrate through the basement membrane and re-attach itself to the matrix and keratinocytes to form a new epidermal melanin unit. ${ }^{7}$

Keratinocytes act on melanocytes by producing several factors that regulate their survival..$^{5}$ They create the necessary microenvironment for the proliferation, differentiation and melanocyte migration. ${ }^{8}$

The fundamental histopathological difference between skin with normal coloration and skin with vitiligo is the absence of functioning melanocytes on the latter. ${ }^{9-12}$ Although there may be viable melanocytes in the altered skin, they are usually absent, which can be verified by Fontana-Masson staining, specific for melanin or by dihydroxyphenyl alanine technique for the demonstration of tyrosinase. ${ }^{13,14}$ Techniques that use autoantibodies to identi- 
fy melanocytic lineage and electron microscopy, also demonstrate that the achromic patches of vitiligo are devoid of melanocytes. ${ }^{15}$ Other stains that may be useful are: DOPA, which detects active melanocytes and HMB-45, Mel-5, NKI/beteb that detect active and inactive melanocytes. ${ }^{16}$

Degenerative alterations in cutaneous nerves and adnexal structures, such as sudoriparous glands, sebaceous glands, or hair follicles, have been identified in old lesions. These alterations were more marked in long-term illnesses, according to an analysis of 74 cases of vitiligo. ${ }^{15-17}$

Inflammatory changes were found more frequently in early lesions. When present, the identified inflammatory cells were mostly CD4+ and CD8+ lymphocytes. ${ }^{17}$ Functionally, these cells obtained from skin with vitiligo can show melanocyte-specific cytotoxicity in non-lesional skin. ${ }^{18}$

In the margins of recent lesions there may be a superficial lymphocytic infiltrate and occasionally, a lichenoid mononuclear infiltrate. At the outer edge of the skin with vitiligo, melanocytes are larger, often vacuolated and with long dendritic processes filled with melanin granules. ${ }^{13}$

Adjacent skin of normal coloration may also present foci of vacuolar alterations in the dermoepidermal junction associated with moderate mononuclear infiltrate. ${ }^{15}$ Infiltration of T cells in the dermoepidermal junction of non-lesional skin was identified in patients with active common vitiligo, followed by the disappearance of melanocytes in the area. ${ }^{19}$ Deposits of extracellular granular material and foci of basal and parabasal keratinocyte vacuolar degeneration were found in electron microscopy analysis of biopsies obtained up to $15 \mathrm{~cm}$ away from vitiligo lesions. ${ }^{20}$

\section{VITILIGO TREATMENT}

The goal of vitiligo treatment is to control the autoimmune damage to melanocytes and stimulate their migration from surrounding skin and adnexal reservoirs. Treatment may be divided into pharmacological, surgical and physical, which can sometimes be combined.

\section{a - Topical \\ b - Systemic \\ 2 - Physical Treatment \\ 3 - Surgical Treatment}

1 - Pharmacological Treatment

\section{1 - Pharmacological Treatment}

a - Topical treatment

\section{Corticosteroids}

Topical corticosteroid therapy is considered a first-line treatment of vitiligo, since it is low-cost and easy to apply. ${ }^{21}$ It is limited by the risk of local adverse effects, such as atrophy, striae and telangiectasias and also systemic side effects. Thus, the use of high-potency topical corticosteroids is more suitable to treat small affected areas, being more effective on the face, elbows and knees, although some authors prefer to use lowpower corticoids on the face and flexural areas. ${ }^{22}$

A meta-analysis demonstrated that class 3 topic corticosteroids had higher efficacy in the treatment of localized vitiligo, compared to class 4 and intralesional corticoids, also showing higher incidence of atrophy in class 4 drugs. ${ }^{23}$

A retrospective study compared the use of high and moderate power topical corticosteroids in 101 children: both groups showed repigmentation in $64 \%$ of cases, stabilization in $24 \%$ and worsening in $11 \%$. In the stratified analysis according to corticosteroid potency, no difference was found between the moderate and high power drugs $(\mathrm{p}=0.03)$, nor in the incidence of local adverse events $(p=0.3)$, However, this study should be interpreted with caution due to variable product dosages, as well as the small number of cases in the group of moderate-power corticoids. ${ }^{21}$

Although studies recommend the use of highpower topical corticosteroids in localized vitiligo, its use should be limited to 2-4 months periods, as lowpower corticosteroids or the use of other immunomodulators should be considered in order to decrease the risk of adverse events. If no clinical response is seen with topical corticosteroids in 3 to 4 months, their application should be suspended. ${ }^{24}$

\section{Calcineurin Inhibitors}

Initially used in transplant patients, calcineurin inhibitors are immunosuppressants, the first of which, cyclosporine, is not used topically due to the lack of good cutaneous absorption. Subsequently, tacrolimus and pimecrolimus, other calcineurin inhibitors, demonstrated good absorption when used topically. ${ }^{25}$

Corticosteroids inhibit collagen synthesis, leading to an increased risk of skin atrophy, especially during prolonged use. An advantage of calcineurin inhibitors is that neocollagenesis does not depend from calcineurin; hence there is no risk of atrophy. ${ }^{25}$

Topical tacrolimus is a calcineurin inhibitor that controls the activity of $\mathrm{T}$ lymphocytes through the inhibition of proinflammatory cytokines, blocking the transcription of the IL-2 genes which are important for the proliferation of cytotoxic T lymphocytes, and also inhibiting the transcription and production of IL-4, IL5, IL-10, IFN- $\gamma$ and TNF- $\alpha .{ }^{25}$ In an open-label, noncomparative study, 42 patients were treated with $0.1 \%$ tacrolimus, twice a day for 6 months, with $76.09 \%$ achieving some degree of repigmentation. Children showed higher response rates than adults and the clin- 
ical forms with best response were vulgar and focal. ${ }^{26}$ In a randomized, double blind study, the use of $0.1 \%$ tacrolimus was as effective as $0.5 \%$ clobetasol propionate, when applied twice a day for 60 days in children ( $p>0.05)$. Both treatments achieve the highest response rates on facial areas or those with high density of hair follicles. Atrophy and telangiectasia were only observed in the clobetasol group. ${ }^{27}$

In a prospective study, $1 \%$ pimecrolimus was compared to $0.5 \%$ clobetasol propionate with results being assessed in lesions on parasagittal planes. The results showed no statistical difference in repigmentation..$^{28}$

The association of a topical immunosuppressive drug with a physical treatment was investigated in a comparative, randomized, single-blinded study that showed better therapeutic response in groups treated by excimer laser $(308 \mathrm{~nm})$ associated with $1 \%$ topical pimecrolimus when compared to LASER used alone. ${ }^{29}$

Calcineurin inhibitors have demonstrated efficacy similar to topical corticosteroids, without the risk of cutaneous atrophy in the long-term use. ${ }^{28}$

\section{Calcipotriol and betamethasone dipropionate}

The use of calcipotriol combined with topical corticosteroids has been reported in the literature. In a case series, a 75\% repigmentation rate was observed in patients that were resistant to previous treatments such as tacrolimus and topic corticosteroids. ${ }^{30,31}$ The use of vitamin D analogues has been associated with narrow-band UVB and Excimer LASER. ${ }^{22}$

\section{Systemic Treatment}

\section{Systemic corticosteroid therapy}

Systemic corticosteroid therapy is used in cases of disseminated vitiligo lesions with rapid progression. Decreased complement-mediated cytotoxicity and levels of antibodies against melanocyte surface have been described in patients with unstable vitiligo responsive to systemic corticosteroids. ${ }^{32}$ A non-comparative study with 81 vitiligo patients treated with prednisolone $0.3 \mathrm{mg} / \mathrm{kg} /$ day for 2 months, progressively reduced until the fifth month, demonstrated control of disease progression and repigmentation in $87.7 \%$ and $74.1 \%$ of cases respectively. ${ }^{32}$

The use of systemic corticosteroids in minipulses has been used in order to minimize adverse events inherent to the treatment. A meta-analysis demonstrated a lower incidence rate of systemic and cutaneous adverse events with the mini-pulse regimen when compared to daily use corticosteroids. ${ }^{23}$

The oral mini-pulse with $5 \mathrm{mg}$ betamethasone/dexamethasone for 2 consecutive days per week was evaluated in patients with extensive vitiligo and/or rapid progression: in 1 to 3 months, $89 \%$ of patients with progressive disease presented lesion stabilization and in 2 to 4 months, $80 \%$ of patients had some degree of repigmentation. ${ }^{33}$

Twenty-nine patients with vitiligo received 2 weekly pulses of $10 \mathrm{mg}$ dexamethasone on 2 consecutive days, for a maximum of 24 weeks, and whilst $88 \%$ achieve progression control, $72.4 \%$ of patients presented no repigmentation. ${ }^{34}$ These studies support the need for further evidence on this form of treatment.

\section{2- Physical Treatment}

Ultraviolet (UV) radiation, both in UVA and UVB spectrum, has been used in the treatment of vitiligo. Its effect is not yet fully understood. It can induce immunosuppression by inhibiting melanocyte destruction or stimulating the increase in their numbers and migratory capacity. ${ }^{35}$

\section{Narrow band UVB - NBUVB}

An effective and safe therapeutic modality, treatment with narrowband UVB $(311 \mathrm{~nm})$ is considered a first-line option for vitiligo. It dispenses the combined use of an oral psoralen, thus freeing patients of ocular and gastrointestinal adverse events related to this drug.

In 1997 a pioneer comparative study, comparing topical PUVA and NBUVB, reported $46 \%$ repigmentation rates in the PUVA group and $67 \%$ in the NBUVB group. The author emphasized the lower cumulative dose in the group undergoing NBUVB and the lower incidence of adverse events. ${ }^{36}$

A double-blind randomized study showed $64 \%$ of patients with more than $50 \%$ repigmentation in the group submitted to NBUVB, compared to $36 \%$ in the group treated with systemic PUVA, evidencing the superiority of NBUVB. ${ }^{37}$

In a preliminary result, 4 cases of vitiligo treated with NBUVB combined with melanotrophic hormone synthetic analog afamelanotide obtained diffuse and fast repigmentation, although more studies are needed. ${ }^{38}$

\section{Phototherapy with UVA and psoralens (PUVA therapy)}

Photochemotherapy is a therapeutic method that consists in the use of a drug that enhances the effects of light. Psoralens are the most commonly used drugs in the treatment of vitiligo, in the forms 8methoxypsoralen, 5-methoxypsoralen or trimethylpsoralen which may be used in their oral and topical presentations..$^{22}$

Introduced in 1948, oral PUVA therapy is a quite popular treatment for vitiligo and involves the application of UVA (320-400nm) and the use of a photosensitizing drug, usually 8 methoxypsoralen administered oral- 
ly 2 hours before light application at a dose of $0.6 \mathrm{mg} / \mathrm{kg}$.

The most frequent short-term adverse events are cutaneous and ocular phototoxicities, nausea and headache while long-term side effects, such as photoaging and increased risk of skin cancer, especially squamous cell carcinoma are dose-related. ${ }^{35}$

Topical PUVA therapy can be used in localized forms of vitiligo in adults and children over 2 years old. Due to the lower doses of UVA used and small absorption of topical psoralen it is considered a safer option.

The better results achieved with NBUVB as well as its greater safety profile when compared to UVA phototherapy, are causing the latter to be less used. ${ }^{35,39}$

\section{Monochromatic Excimer light}

The combination of monochromatic excimer light with xenon chloride gas emits light with a wavelength of $308 \mathrm{~nm}$. There are two forms of producing this light; the excimer LASER that produces a coherent and monochromatic light and the excimer lamp that produces a non-directional and non-coherent light of $308 \mathrm{~nm}$. These treatment forms differ from NBUVB in their mode of application, as they may be applied in a more localized fashion in the lesions.

Two comparative studies in patients with symmetrically distributed vitiligo lesions, compared the response between parasagittal planes treated by excimer laser and NBUVB, showing quicker response and larger area of repigmentation in the excimer group..$^{40,41}$

Studies comparing excimer laser versus lamps found no difference in response rates, although lamps are more time consuming to deliver the required dose, and this period can be quite long in patients with disseminated lesions. ${ }^{42-44}$

\section{3 - Surgical therapy}

Surgical melanocyte transplantation is an important therapeutic option available for patients with stable disease who failed to respond to classical therapies. ${ }^{45}$ It is indicated even for traditionally refractory areas such as distal extremities, elbows, knees, nipples, eyelids and lips. ${ }^{46}$ Furthermore, the appropriate choice of patients, with the exclusion of those presenting Koebner phenomenon and active disease is essential to prevent achromic lesions in the donor areas and achieve better results in the receiving areas. ${ }^{47} \mathrm{~A}$ minigrafting test can be performed previously if there are doubts concerning the stability of the disease. ${ }^{48}$

Punch Grafting (PG) is the easiest and lowest cost technique, although it is generally limited to treating small areas. ${ }^{49}$ The recipient area is prepared by performing multiple punches of equal size or 0.25 to 0.5 $\mathrm{mm}$ smaller than those extracted from the donor area. ${ }^{46}$
Larger grafts often produce a cosmetically undesirable effect, known as cobblestoning (cobblestone appearance). This side effect usually resolves spontaneously or with treatments like electrofulguration. ${ }^{50}$ Punch grafting is able to produce excellent repigmentation and good cosmetic results. In a prospective study with a large number of patients, $74.55 \%$ of those undergoing PG achieved $90-100 \%$ repigmentation. ${ }^{50}$ Evidences also suggest that the association of PG with phototherapy (narrowband UVB) and topical corticosteroids may enhance therapeutic results for this technique. ${ }^{51,52}$

Suction blister epidermal grafting (SBEG) is a technique for obtaining thin skin grafts through dermoepidermal separation caused by prolonged suction applied in the donor area. The recipient area is prepared by dermabrasion or laser application (Erbium:YAG or $\left.\mathrm{CO}_{2}\right) \cdot{ }^{48}$ Although SBEG is a time consuming technique restricted to treating small areas, it can provide good therapeutic results (repigmentation $>75 \%$ in $89 \%$ of patients in a recent study) with minimal chances of producing unsightly scars in the donor area. ${ }^{50}$

Split-thickness skin grafts also have good repigmentation rates, $90-100 \%$ according to Agarwal et al and also the advantage of treating large areas with a single procedure. The graft is obtained with the aid of a dermatome, although this procedure requires skill and experience..$^{53}$ Furthermore, the incompatibility of colors in the receiving area and scarring in the donor area are potential side effects of this technique. ${ }^{48}$

Autologous non-cultured epidermal cell suspension (NCECS) grafting, a cellular transplantation technique, has the advantage of treating a receiving area about 10 times larger than the donor area, with excellent color compatibility. ${ }^{54}$ In this procedure, a skin fragment is obtained by shaving biopsy and the epidermis is separated from the dermis using a trypsin solution. After several additional steps, a suspension of keratinocytes and melanocytes is obtained and transplanted to the scarified skin in the receiver area. ${ }^{39,48,54}$ In a randomized study with 41 patients, repigmentation was considered excellent (90-100\% repigmentation) in $71 \%$ of lesions in the NCECS group, compared with $27 \%$ of lesions on the SBEG technique group. ${ }^{54}$ Cell culture has also been used as a means of increasing the number of viable cells and treat even larger areas using less donor tissue. However, this method is costly and requires the support of a tissue culture laboratory. ${ }^{48}$ Furthermore, the use of some mitogens present in the culture medium raised questions about the safety of the procedure. ${ }^{55}$

\section{FINAL CONSIDERATIONS}

Much has been discovered in recent years about the pathophysiology of the disease, pointing clinical findings to three main factors: 1) adhesion deficit 
throughout the epidermis, not only affecting the adhesion of melanocytes, but also keratinocytes; 2) increased local oxidative stress; 3 ) T lymphocyte-mediated cytotoxicity against melanocytes; however, the real contribution of each of these factors for each individual patient is still subject of much investigation.

After decades without a proper global joint effort, an article from the consensus on vitiligo classification was finally published, nevertheless, there are still some subtypes of vitiligo that have not been properly defined or positioned in the type classification, which should be subject to a new classification review in the future, especially associating the characterization of clinical types and subtypes with moleculargenetic tests.

Among the major challenges of modern epidemiological genetics is the validation of the association findings, through independent replication studies and, ultimately, functional studies that will explain the actual causative biological effect of the association or link observed. The task is far from trivial: beyond the classic question of establishing a cause-effect relationship starting from an association finding, the sce- nario that is currently emerging from large-scale studies of complex genetic characteristics (such as genome-wide association studies or GWAS) is even more challenging. For example, it is possible that the genetic control component of diseases such as vitiligo, described in observational studies, is pulverized among dozens - perhaps hundreds - of genes, each contributing with a small portion of the final relative risk. It is yet possible that genetic variants, very rare at the population level but found throughout the genome, are responsible for a large portion of the said relative risk - testing this hypothesis will require the ability to sequence the entire human genome, only feasible to very large laboratories recently. Finally, it is possible that the clinical phenotypes with which we classically work, such as "vitiligo", are too complex in this case, the definition of more controlled molecular phenotypes defined from in vitro biological systems, for example, can be pivotal. Developments in this direction are starting to become public, opening new approaches toward the full understanding of the genetic basis of complex diseases in general and especially of vitiligo.

\section{REFERENCES}

1. Ezzedine K, Lim HW, Suzuki T, Katayama I, Hamzavi I, Lan CC, et al. Revised classification/nomenclature of vitiligo and related issues: the Vitiligo Global Issues Consensus Conference. Pigment Cell Melanoma Res. 2012;25:E1-13.

2. Silva de Castro CC, do Nascimento LM, Olandoski M, Mira MT. A pattern of association between clinical form of vitiligo and disease-related variables in a Brazilian population. J Dermatol Sci. 2012;65:63-7.

3. Lin JY, Fisher DE. Melanocyte biology and skin pigmentation. Nature. 2007;445:843-50.

4. Westerhof W. The discovery of the human melanocyte. Pigment Cell Res. 2006;19:183-93.

5. Lee AY, Kim NH, Choi WI, Youm YH. Less keratinocyte-derived factors related to more keratinocyte apoptosis in depigmented than normally pigmented suctionblistered epidermis may cause passive melanocyte death in vitiligo. J Invest Dermatol. 2005;124:976-83.

6. Fitzpatrick TB. Dermatology in general medicine. 4th ed. New York: McGraw-Hill; 1993.

7. Haass NK, Herlyn M. Normal human melanocyte homeostasis as a paradigm for understanding melanoma. J Investig Dermatol Symp Proc. 2005;10:153-63.

8. Carlson JA, Linette GP, Aplin A, Ng B, Slominski A. Melanocyte receptors: clinical implications and therapeutic relevance. Dermatol Clin. 2007;25:541-57, viii-ix.

9. Moretti S, Spallanzani A, Amato L, Hautmann G, Gallerani I, Fabiani M, et al. New insights into the pathogenesis of vitiligo: imbalance of epidermal cytokines at sites of lesions. Pigment Cell Res. 2002;15:87-92.

10. Le Poole IC, Das PK. Microscopic changes in vitiligo. Clin Dermatol. 1997;15:863-73.

11. Le Poole IC, van den Wijngaard RM, Westerhof W, Dutrieux RP, Das PK. Presence or absence of melanocytes in vitiligo lesions: an immunohistochemical investigation. J Invest Dermatol. 1993;100:816-22.

12. Tobin DJ, Swanson NN, Pittelkow MR, Peters EM, Schallreuter KU. Melanocytes are not absent in lesional skin of long duration vitiligo. J Pathol. 2000;191:407-16.

13. Yaghoobi R, Omidian M, Bagherani N. Vitiligo: a review of the published work. J Dermatol. 2011;38:419-31.

14. Kim YC, Kim YJ, Kang HY, Sohn S, Lee ES. Histopathologic features in vitiligo. Am J Dermatopathol. 2008;30:112-6.

15. Yaghoobi R, Razi T, Feily A. Clinical image: an unusual pigmented basal cell carcinoma arising from vulva. Acta Dermatovenerol Alp Panonica Adriat. 2011;20:81-2.
16. Alikhan A, Felsten LM, Daly M, Petronic-Rosic V. Vitiligo: a comprehensive overview Part I. Introduction, epidemiology, quality of life, diagnosis, differential diagnosis, associations, histopathology, etiology, and work-up. J Am Acad Dermatol. 2011;65:473-91.

17. Boissy RE, Dell'Anna ML, Picardo M. On the pathophysiology of vitiligo: possible treatment options. Indian J Dermatol Venereol Leprol. 2012;78:24-9.

18. van den Boorn JG, Konijnenberg D, Dellemijn TA, van der Veen JP, Bos JD, Melief $\mathrm{CJ}$, et al. Autoimmune destruction of skin melanocytes by perilesional T cells from vitiligo patients. J Invest Dermatol. 2009;129:2220-32.

19. Wańkowicz-Kalińska A, van den Wijngaard RM, Tigges BJ, Westerhof W, Ogg GS, Cerundolo V, et al. Immunopolarization of CD4+ and CD8+ T cells to Type-1-like is associated with melanocyte loss in human vitiligo. Lab Invest. 2003;83:683-95.

20. Moellmann G, Klein-Angerer S, Scollay DA, Nordlund JJ, Lerner AB. Extracellular granular material and degeneration of keratinocytes in the normally pigmented epidermis of patients with vitiligo. J Invest Dermatol. 1982;79:321-30.

21. Kwinter J, Pelletier J, Khambalia A, Pope E. High-potency steroid use in children with vitiligo: a retrospective study. J Am Acad Dermatol. 2007;56:236-41.

22. Lotti T, Berti S, Moretti S. Vitiligo therapy. Expert Opin Pharmacother. 2009;10:2779-85.

23. Njoo MD, Spuls PI, Bos JD, Westerhof W, Bossuyt PM. Nonsurgical repigmentation therapies in vitiligo. Meta-analysis of the literature. Arch Dermatol. 1998;134:1532-40.

24. Falabella R, Barona MI. Update on skin repigmentation therapies in vitiligo. Pigment Cell Melanoma Res. 2009;22:42-65.

25. Kostovic K, Pasic A. New treatment modalities for vitiligo: focus on topical immunomodulators. Drugs. 2005;65:447-59.

26. Udompataikul M, Boonsupthip P, Siriwattanagate R. Effectiveness of $0.1 \%$ topical tacrolimus in adult and children patients with vitiligo. J Dermatol. 2011;38:536-40.

27. Lepe V, Moncada B, Castanedo-Cazares JP, Torres-Alvarez MB, Ortiz CA, TorresRubalcava $A B$. A double-blind randomized trial of $0.1 \%$ tacrolimus vs $0.05 \%$ clobetasol for the treatment of childhood vitiligo. Arch Dermatol. 2003;139:581-5.

28. Coskun B, Saral Y, Turgut D. Topical $0.05 \%$ clobetasol propionate versus $1 \%$ pimecrolimus ointment in vitiligo. Eur J Dermatol. 2005;15:88-91. 
29. Hui-Lan Y, Xiao-Yan H, Jian-Yong F, Zong-Rong L. Combination of 308-nm excimer laser with topical pimecrolimus for the treatment of childhood vitiligo. Pediatr Dermatol. 2009;26:354-6.

30. Travis LB, Silverberg NB. Calcipotriene and corticosteroid combination therapy for vitiligo. Pediatr Dermatol. 2004;21:495-8.

31. Newman MD, Silverberg NB. Once-daily application of calcipotriene $0.005 \%$-betamethasone dipropionate $0.064 \%$ ointment for repigmentation of facial vitiligo. Cutis. 2011;88:256-9.

32. Kim SM, Lee HS, Hann SK. The efficacy of low-dose oral corticosteroids in the treatment of vitiligo patients. Int J Dermatol. 1999;38:546-50.

33. Pasricha JS1, Khaitan BK. Oral mini-pulse therapy with betamethasone in vitiligo patients having extensive or fast-spreading disease. Int J Dermatol. 1993;32:753-7.

34. Radakovic-Fijan S, Fürnsinn-Friedl AM, Hönigsmann H, Tanew A. Oral dexamethasone pulse treatment for vitiligo. J Am Acad Dermatol. 2001;44:814-7.

35. Pacifico A, Leone G. Photo(chemo)therapy for vitiligo. Photodermatol Photoimmunol Photomed. 2011;27:261-77.

36. Westerhof W, Nieuweboer-Krobotova L. Treatment of vitiligo with UV-B radiation vs topical psoralen plus UV-A. Arch Dermatol. 1997;133:1525-8.

37. Yones SS, Palmer RA, Garibaldinos TM, Hawk JL. Randomized double-blind trial of treatment of vitiligo: efficacy of psoralen-UV-A therapy vs Narrowband-UV-B therapy. Arch Dermatol. 2007;143:578-84.

38. Grimes PE, Hamzavi I, Lebwohl M, Ortonne JP, Lim HW. The efficacy of afamelanotide and narrowband UV-B phototherapy for repigmentation of vitiligo. JAMA Dermatol. 2013;149:68-73.

39. Felsten LM, Alikhan A, Petronic-Rosic V. Vitiligo: a comprehensive overview Part II: treatment options and approach to treatment. J Am Acad Dermatol. 2011;65:493-514

40. Hong SB, Park HH, Lee MH. Short-term effects of 308-nm xenon-chloride excimer laser and narrow-band ultraviolet $B$ in the treatment of vitiligo: a comparative study. J Korean Med Sci. 2005;20:273-8.

41. Casacci M1, Thomas P, Pacifico A, Bonnevalle A, Paro Vidolin A, Leone G. Comparison between 308-nm monochromatic excimer light and narrowband UVB phototherapy (311-313 nm) in the treatment of vitiligo--a multicentre controlled study. J Eur Acad Dermatol Venereol. 2007;21:956-63.

42. Le Duff F, Fontas E, Giacchero D, Sillard L, Lacour JP, Ortonne JP, Passeron T. et al. 308-nm excimer lamp vs. 308-nm excimer laser for treating vitiligo: a randomized study. Br J Dermatol. 2010;163:188-92.

43. Shi Q, Li K, Fu J, Wang Y, Ma C, Li Q, et al. Comparison of the 308-nm excimer laser with the 308-nm excimer lamp in the treatment of vitiligo--a randomized bilateral comparison study. Photodermatol Photoimmunol Photomed. 2013;29:27-33.

44. Park KK, Liao W, Murase JE. A review of monochromatic excimer light in vitiligo. Br J Dermatol. 2012;167:468-78.

45. Njoo MD, Westerhof W, Bos JD, Bossuyt PM. A systematic review of autologous transplantation methods in vitiligo. Arch Dermatol. 1998;134:1543-9.

46. Patel NS, Paghdal KV, Cohen GF. Advanced treatment modalities for vitiligo. Dermatol Surg. 2012;38:381-91.

47. Fongers A, Wolkerstorfer A, Nieuweboer-Krobotova L, Krawczyk P, Tóth GG, van der Veen JP. Long-term results of 2-mm punch grafting in patients with vitiligo vulgaris and segmental vitiligo: effect of disease activity. $\mathrm{Br} J$ Dermatol. 2009;161:1105-11.

48. Parsad D, Gupta S; IADVL Dermatosurgery Task Force. Standard guidelines of care for vitiligo surgery. Indian J Dermatol Venereol Leprol. 2008;74:S37-45.
49. Taieb A, Alomar A, Böhm M, Dell'anna ML, De Pase A, Eleftheriadou V, et al. Guidelines for the management of vitiligo: the European Dermatology Forum consensus. Br J Dermatol. 2013;168:5-19.

50. Falabella R. Surgical treatment of vitiligo: why, when and how. J Eur Acad Dermatol Venereol. 2003;17:518-20.

51. Lahiri K, Malakar S, Sarma N, Banerjee U. Repigmentation of vitiligo with punch grafting and narrow-band UV-B (311 nm)--a prospective study. Int J Dermatol. 2006;45:649-55.

52. Saldanha KD, Machado Filho CD, Paschoal FM. Action of topical mometasone on the pigmented halos of micrografting in patients with vitiligo. An Bras Dermatol. 2012;87:685-90.

53. Agrawal K, Agrawal A. Vitiligo: repigmentation with dermabrasion and thin splitthickness skin graft. Dermatol Surg. 1995;21:295-300.

54. Budania A, Parsad D, Kanwar AJ, Dogra S. Comparison between autologous noncultured epidermal cell suspension and suction blister epidermal grafting in stable vitiligo: a randomized study. Br J Dermatol. 2012;167:1295-301.

55. Czajkowski R, Pokrywczynska M, Placek W, Zegarska B, Tadrowski T, Drewa T. Transplantation of cultured autologous melanocytes: hope or danger? Cell Transplant. 2010;19:639-43.

\author{
MAILING ADDRESS: \\ Caio Cesar Silva de Castro \\ Praça Rui Barbosa, 245 \\ 80010-030 - Curitiba - PR \\ Brazil \\ E-mail: caio.castro@pucpr.br
}

How to cite this article: Reichert-Faria A, Tarlé RG, Dellatorre G, Mira MT, Silva de Castro CC. Vitiligo - Part 2 classification, histopathology and treatment. An Bras Dermatol. 2014;89(5):784-90. 